Check for updates

Cite this: Nanoscale Adv., 2019, 1, 314

\title{
Interfacial complexation driven three-dimensional assembly of cationic phosphorus dendrimers and graphene oxide sheets $\dagger$
}

\author{
Nadia Katir, ${ }^{* a}$ Anass Benayad, ${ }^{\text {b }}$ Denis Rouchon, ${ }^{c}$ Nathalie Marcotte, ${ }^{d}$ Nabil El \\ Brahmi, ${ }^{a}$ Jean Pierre Majoral, ${ }^{e}$ Mosto Bousmina ${ }^{a}$ and Abdelkrim El Kadib*a
}

\begin{abstract}
High content nitrogen, sulfur and phosphorus heteroatoms assembled in tree-like dendrimers $\left(\mathrm{DG}_{n}\right)$ are confined within the galleries of two-dimensional graphene oxide (GO). The presence of the ternary diethyl- $N$-ethyl-ammonium groups on the dendrimer peripheries ensures the exfoliation of graphene sheets thereby affording interfacially bridged, three-dimensional heteroatom-enriched graphene-based hybrid nanostructures ( $D G_{n}-G O$ ). Dendrimer generation (from 1 to 4 ) that reflects the bulkiness of these conceived nano-trees impacts increasingly the degree of dispersion-exfoliation and sheet desordering. The long-term stability of these aqueous suspensions associated with their handling flexibility allows uniform accommodation of the resulting hybrid materials as flame-retardants in bioplastics.
\end{abstract}

Received 18th June 2018

Accepted 22nd June 2018

DOI: $10.1039 / c 8 n a 00047 f$

rsc.li/nanoscale-advances

more relevant to produce high-quality graphene materials but

\section{Introduction}

Peeling-off tiny graphene sheets, reported fourteen years ago, ${ }^{1}$ have initiated an avalanche of investigations primarily focused on their massive production, ${ }^{2}$ surface-functionalisation, ${ }^{3}$ nanocomposite manufacturing ${ }^{4}$ and their subsequent use for energy storage and conversion, ${ }^{5}$ catalysis, ${ }^{6}$ gas sensing, ${ }^{7}$ and in nanomedicine and healthcare applications. ${ }^{8}$ Owing to their fascinating properties, graphene-based materials stand as prominent contenders for future emerging nanotechnologies, specifically those tightly connected to flexible and wearable electronics.

Splitting tactoids or layered graphite stacks into individual, well-exposed thin carbon sheets is a fundamental prerequisite to access high-quality graphene materials. ${ }^{9}$ The introduction of heteroatoms within these individual sheets by insertion, impregnation or covalent grafting allows for modulating further their physical and chemical properties. ${ }^{3}$ Physical methods are

${ }^{a}$ Euromed Research Center, Engineering Division, Euro-Mediterranean University of Fez (UEMF), Route de Meknes, 30000, Fès, Morocco. E-mail: a.elkadib@ueuromed.org ${ }^{b}$ CEA, LITEN, Department of Nanomaterials, MINATEC, 17 rue des martyrs, F-38054 Grenoble Cedex 09, France

${ }^{c}$ CEA, LETI, Department of Nanomaterials, Minatec Campus, F-38054 Grenoble Cedex 09, France

${ }^{d}$ Institut Charles Gerhardt UMR 5253, CNRS/ENSCM/UM, 8 rue de l'Ecole Normale, Montpellier F-34295 Cedex, France

${ }^{e}$ Laboratoire de Chimie de Coordination (LCC) CNRS, 205 route de Narbonne, 31077 Toulouse, France

$\dagger$ Electronic supplementary information (ESI) available: Synthesis and characterisation of dendrimers, NMR spectra, DRIFT, XRD, UV-visible, SEM, EDX, TEM, XPS, TGA, and RAMAN analysis of materials. See DOI: 10.1039/c8na00047f are not, unfortunately, cost-effective because of the need for sophisticated apparatus and energy-associated procedures. Oppositely, the straightforwardness and versatility of liquidphase synthesis provide a large library of chemically modified graphene materials.

Graphene oxide (GO), obtained by oxidation of graphite, is prone to dispersion in aqueous and liquid solvents and to chemical modification owing to the presence of polar, transformable oxygenated epoxides, alcohols, ketones and carboxylic acids. ${ }^{10}$ Beyond its molecular-surface functionalisation, more sophisticated nanostructures including graphene oxide coated polymers and biopolymers, liposomes, DNA, polyoxometalate and other assembled graphene-based nanomaterials have been recently reported. ${ }^{11}$ This hybridisation at the nanoscale brings novel reactivity to graphene sheets and substantially enhances their long-term dispersion and stability. Despite these achievements, further innovative investigations are necessary to assemble these tiny building-blocks into a rationally designed, functional three-dimensional architecture suitable for practical applications.

Having recently revealed many specific behaviours of dendrimers in materials science, ${ }^{12}$ we envisioned that their supramolecular association with graphene sheets could provide a novo-design concept for new functional hybrid materials. ${ }^{13}$ Dendrimers differ from their linear polymer congeners by their defined size, shape and topology, molecular diversity from the core to the surface, tunable flexibility or rigidity depending on the spacer installed in their branching units, amphiphilicity, and their well-established "generation effect". ${ }^{14}$ More specifically, phosphorus dendrimers display some intrinsic features including their organic-inorganic character, their typically 
hydrophobic cylcotriphosphazene core, and the high content of nitrogen, sulfur and phosphorus heteroatoms in their framework that impart them with excellent stability. ${ }^{15}$ The presence of the phosphorus element allows accurate elucidation of the chemical functionalisation undertaken on the outer-sphere of these architectures. ${ }^{\mathbf{1 6}}$ Their ability to swell in polar solvents ${ }^{\mathbf{1 7}}$ provides access to hierarchically porous pockets that entrap enzymes and living-cells, ${ }^{18}$ confine and stabilize nanoparticles ${ }^{\mathbf{1 9}}$ and prematurely convert amorphous bursts into discrete mesocrystals. ${ }^{12,20}$ In a conventional medium, this latter phenomenon occurs commonly in the solid state under high temperature conditions.

Based on the aforementioned achievements and our own endeavours, we became interested in the secondary-structure of phosphorus-dendrimers as a potent efficient exfoliating medium and heteroatom provider for graphene materials. We herein report the liquid-exfoliation of graphene oxide by a set of terminated - ternary diethyl- $N$-ethyl-ammonium - phosphorus dendrimers. The choice of peripheral ammonium groups was dictated by their favourable interaction with epoxy, alcohol and carboxylic acid, which efficiently stabilize exfoliated sheets. ${ }^{21}$ The assembled dendrimer-graphene oxide hybrid materials thus obtained were isolated and characterized. Their stability was disclosed and their reactivity with hydrazine as a reducing agent was also undertaken with a special emphasis to elucidate the chemical interactions occurring at the material interface. We also present the wrapping of these exfoliated sheets in a biobased shell-waste polysaccharide for the preparation of thermally stable, functional phosphorus-based hybrid bioplastics.

\section{Results and discussion}

\section{Preparation of $\mathrm{DG}_{\boldsymbol{n}}$-GO}

In our experiments, four different generations of cationic phosphorhydrazone dendrimers, denoted as $\mathbf{D G}_{\boldsymbol{n}}(n=1-4)$, were used. ${ }^{22}$ These dendrimers feature similar core (cyclotriphosphazene) and similar peripheral groups (ternary diethyl$\mathrm{N}$-ethyl-ammonium) but differ in their size (number of phosphorus layers) and the number of ternary ammonium groups (12 ternary ammoniums for $\mathbf{D G}_{\mathbf{1}}, 24$ for $\mathbf{D G}_{\mathbf{2}}, 48$ for $\mathbf{D G}_{\mathbf{3}}$ and 96 for $\mathbf{D G}_{\mathbf{4}}$ ) (Fig. 1a).

Liquid phase exfoliation was performed by subjecting $40 \mathrm{mg}$ of graphene oxide (GO) in $100 \mathrm{~mL}$ of water to sonication. A ternary diethyl- $N$-ethyl-ammonium-terminated dendrimer $\mathbf{D G}_{\boldsymbol{n}}$ was then added to the solution and the mixture was stirred for 24 hours at room temperature (see the Experimental section for details). It is noteworthy that immediate flocculation was observed with a significant expansion of the solution volume, typical of the interfacial polyionic complexation between the nanosheets and the cationic dendrimers. ${ }^{23}$ The solution was then filtered by centrifugation and the precipitate was washed four times with water to remove any soluble, unreacted dendrimers before drying the resulting $\mathbf{D G}_{\boldsymbol{n}}$-GO materials under vacuum at room temperature.

DRIFT analysis of the resulting dark green powder reveals a typical fingerprint of cationic phosphorus dendrimers (Fig. S2, ESI $\dagger$ ). Accurate details were drawn from solid-state NMR spectroscopy. While CP MAS ${ }^{13} \mathrm{C}$ NMR of the starting GO displays only three signals nearly at 59, 69 and $132 \mathrm{ppm}$, attributed respectively to $\mathrm{C}-\mathrm{O}-\mathrm{C}, \mathrm{CH}_{2}-\mathrm{OH}$ and $\mathrm{C}=\mathrm{C}$, ${ }^{24}$ wellresolved signals assignable to the dendritic skeleton are also observed for $\mathbf{D G}_{\boldsymbol{n}}$-GO hybrid materials. The characteristic methylene appears at $8.7 \mathrm{ppm}$, the $N$-methylated one at $33 \mathrm{ppm}$, the adjacent $\mathrm{CH}_{2}-\mathrm{CH}_{3}$ at $46 \mathrm{ppm}$ and the aromatics between 122 and 133 ppm. A complete disappearance of the signal attributed to the epoxide groups in GO (at $\sim 59$ to $60 \mathrm{ppm}$ ) was observed, pointing out to the ring opening of the latter by nucleophilic species belonging to $\mathbf{D G}_{n} \cdot{ }^{24}$ A slight shift to high values was also observed for the signal belonging to graphenic $\mathrm{CH}_{2}-\mathrm{OH}$ from 68 to 71 ppm (Fig. S3a, ESI $\dagger$ ), owing much probably to the proximal presence of heteroatoms or dynamic sliding of $\mathbf{D G}_{\boldsymbol{n}}$ at the carbon layers. ${ }^{24} \mathrm{CP}$ MAS ${ }^{31} \mathrm{P}$ NMR allows discriminating signals of the phosphorus core $(\sim 5$ to $8 \mathrm{ppm})$ and the ones installed within the branches ( $\sim 67$ to $70 \mathrm{ppm})$. The $P^{\text {core }}$ versus $P^{\text {branch }}$ intensity in $\mathbf{D G}_{\boldsymbol{n}}$-GO was inverted by increasing the dendrimer generation, consistent with their molecular structures (Fig. S3b, ESI $\dagger$ ). X-ray diffraction (XRD) of the starting GO reveals a signal at a $2 \theta$ value of $9.68^{\circ}$ that reflects the basal distance $(d=0.91 \mathrm{~nm})$ of the periodic layers. A shift to $6.81^{\circ}$ was observed once subjected to $\mathbf{D G}_{\mathbf{1}}$ (the smallest, first generation phosphorus dendrimer), indicating a significant expansion of the galleries to $d=1.29 \mathrm{~nm}$ because of the intercalation of the dendrimer. No signal was observed for more bulky $\mathbf{D G}_{2}, \mathbf{D G}_{3}$ and $\mathbf{D G}_{4}$, indicating complete delamination of the GO layers (Fig. S4, ESI $\dagger$ ). During sonication, the high shear impact allows for expanding the basal distance where the adsorbed dendrimer on the graphitic surface constitutes the driving force for preventing the restacking of graphene sheets. As the process goes on, the strong chemical interactions occurring at the dendrimer-graphene oxide interface reduce the motion of sheets and provide enough stability to resist the capillary forces acting on their framework during water removal.

SEM and TEM analyses were undertaken to visualise these objects in the micro- and nano-scale domain. SEM reveals crumpled layers that are connected to each other to form continuous stacks of several tens of nanometers with smooth compact layers inside (Fig. 2a, b and S5a, ESI $\dagger$ ). This aligned densification at the microscale can be rooted in the occurrence of both physical and chemical cross-linking between cationic dendrimers and negative GO sheets. Individual, separated or uncovered graphene layers are not observed indicating that in principle, all sheets are entangled by phosphorus dendrimers, resulting in three-dimensional hybrid materials. EDX mapping confirms the presence of phosphorus, nitrogen, and sulphur supplied from the starting dendrimers and their homogenous distribution through the whole material network (Fig. 2c). TEM analyses show that $\mathbf{D G}_{\boldsymbol{n}}$-GO materials are built from transparent thin layers where the intimate self-assembly of the two buildingblocks affords wrinkled and crumpled nanostructures (Fig. 2d). The presence of aligned tubular layers $(300 \mathrm{~nm}$ to $2 \mu \mathrm{m}$ in length and 8 to $12 \mathrm{~nm}$ in width) as revealed by HRTEM analysis indicates an extension of the self-assembly mechanism beyond the nanoscale level (Fig. 2e, f and S5b, ESI $\dagger$ ). This result finds its 
a

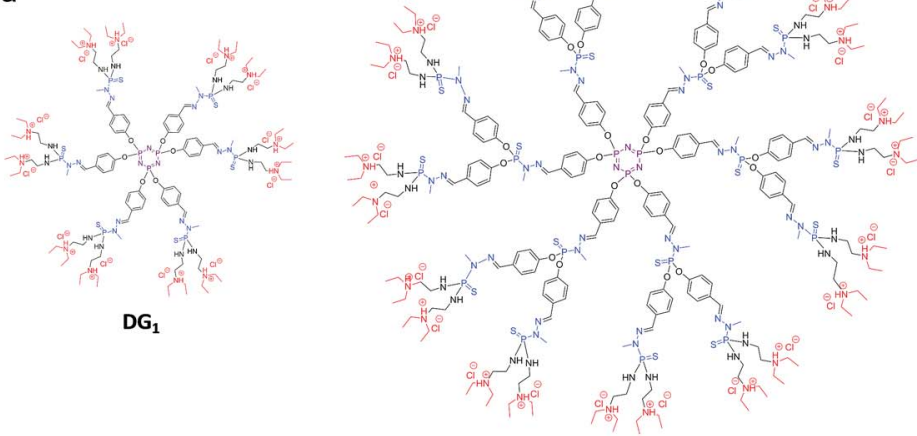

$\mathrm{DG}_{2}$

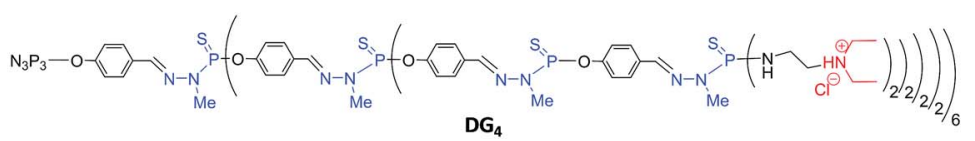

b

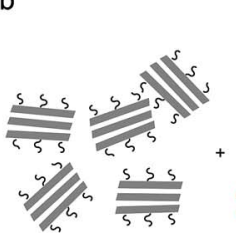

6о

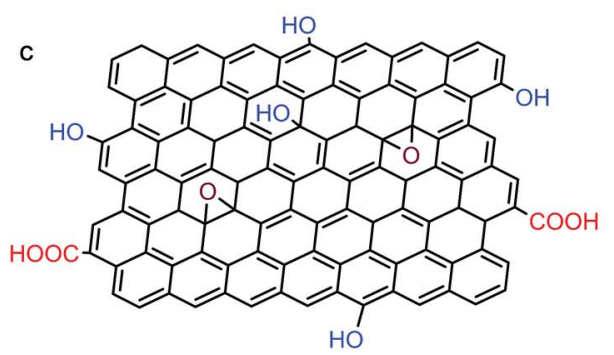

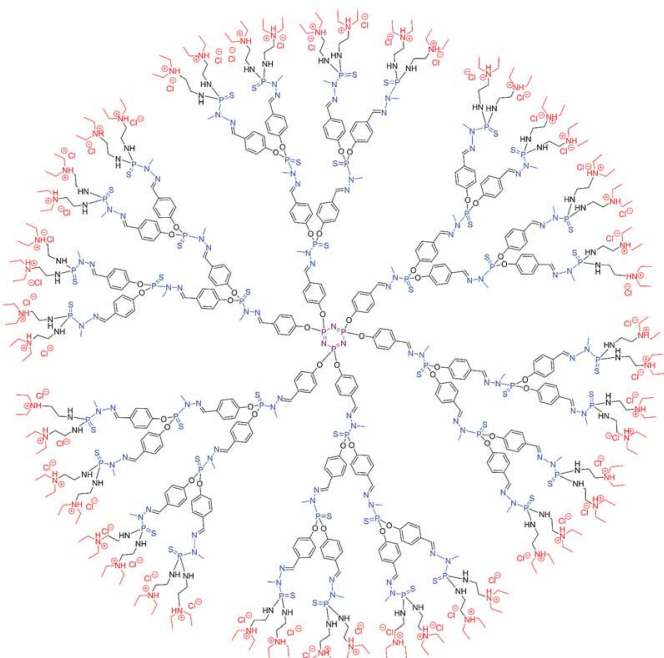

$\mathrm{DG}_{3}$

Fig. 1 (a) Chemical structures of dendrimers $D G_{n}$. (b) Illustration of the exfoliation of layered graphitic oxide materials by ammonium-terminated phosphorus dendrimers and their subsequent reduction. (c) Structures of $G O$. (d) Digital photos of $D G_{1}-G O$ and $D G_{1}-R G O$ dispersion in aqueous solution.
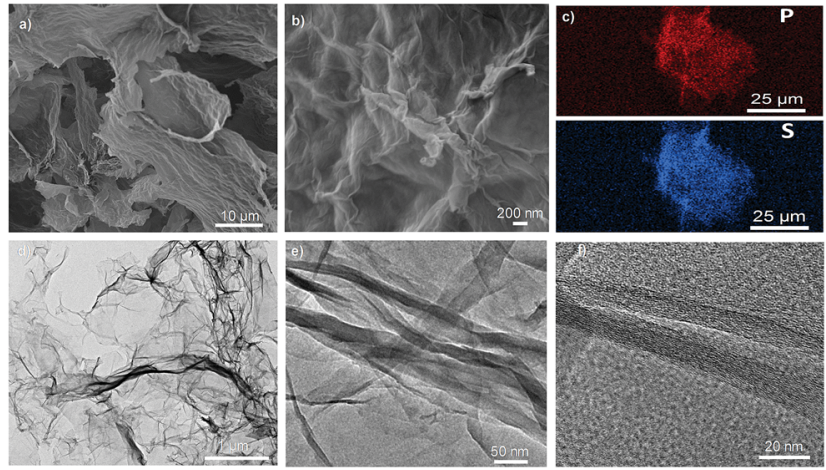

Fig. 2 Microscopic analyses of $\mathrm{DG}_{4}-\mathrm{GO}$. (a and b) SEM of $\mathrm{DG}_{4}-\mathrm{GO}$. (c) EDX mapping of phosphorus (up) and sulfur (bottom). (d) TEM and (e and f) HRTEM of $\mathrm{DG}_{4}-\mathrm{GO}$. root in the dual role of the cationic dendrimer that acts both as an exfoliating medium and as a cross-linking agent owing to the presence of abundant reticular anchoring points on its periphery. A quite similar pattern was previously reported by assembling polyethylenimine and graphene oxide nanoarchitectures. ${ }^{25}$

The resulting solid materials can be re-dispersed in water and the precipitation-dispersion process can be repeated several times without graphene sedimentation or dendrimer leaching. UV-visible analyses of the suspended $\mathbf{D G}_{\boldsymbol{n}}$-GO hybrids display a holistic bond with maxima at 286-290 $\mathrm{nm}$ attributed to the corresponding dendrimer, with a tail extending to the visible region due to the presence of conjugated aromatics belonging to graphene oxide sheets (Fig. S6, ESI $\dagger$ ). ${ }^{24}$ Compared to native $\mathbf{D G}_{\boldsymbol{n}}$ that displayed a wavelength maximum at $285 \mathrm{~nm}$, 
DG $_{\boldsymbol{n}}$-GO maxima of these materials were shifted to higher values with a significant broadening of the band that may indicate the presence of both covalent and physical interactions as well as free terminal groups within the dendrimer skeleton.

A significant improvement of the thermal stability was noticed for $\mathbf{D G}_{\boldsymbol{n}}$-GO compared to the pristine $\mathbf{G O}$ as illustrated by their TGA profiles. For instance, the $T_{40}$ corresponding to the loss of $40 \mathrm{wt} \%$ was shifted from $254{ }^{\circ} \mathrm{C}$ for $\mathbf{G O}$ to around $480{ }^{\circ} \mathrm{C}$ for $\mathbf{D G}_{\mathbf{1}}-\mathbf{G O}, \mathbf{D G}_{\mathbf{2}}-\mathbf{G O}$ and $\mathbf{D G}_{\mathbf{3}}$-GO. An impressive $T_{40}$ of $502{ }^{\circ} \mathrm{C}$ was reached for $\mathbf{D G}_{\mathbf{4}}$-GO (Fig. S11, ESI $\dagger$ ).

\section{Preparation of $\mathrm{DG}_{\boldsymbol{n}}-\mathrm{RGO}$}

Having ascertained the exfoliation of graphene oxide sheets by phosphorus dendrimers and the tight presence of the latter within the tectonic nanostructure, one important question was how the presence of phosphorus dendrimers affects the accessibility to the graphene surface during reduction. Although complete restoration of graphene from graphene oxide is quite impossible (even under forcing conditions), ${ }^{\mathbf{1 0 2 6}}$ reduced graphene oxide (RGO) displayed improved electrical conductivity and thermal and chemical stability, making the corresponding hybrid materials of strong interest. ${ }^{27}$ With this in mind, we subjected $\mathbf{D G}_{\boldsymbol{n}}$-GO aqueous suspensions to hydrazine treatment followed by filtration and extensive washing before drying the resulting solid materials under vacuum. At this stage, two experimental observations must be noted: (i) while the initial exfoliation step did not affect the dark green colour of the starting graphene oxide, a dramatic change to a more pronounced black material was noticed during hydrazine treatment; (ii) simultaneously, while $\mathbf{D G}_{\boldsymbol{n}}$-GO flocculated in solution, significant volume shrinkage was observed during hydrazine treatment, strongly suggesting an alteration of the ionic interfacial complexation of the two building blocks. Similar to their parent materials, the resulting $\mathbf{D G}_{\boldsymbol{n}}$-RGO solids can be isolated and re-dispersed in water with good temporal stability. The precipitation-dispersion cycle can be repeated at least five times without leaching of the dendrimer skeleton from the graphenic surface material. It should be noted that $\mathbf{D G}_{\mathbf{1}}$ RGO provides the less-stable dispersion among the $\mathbf{D G}_{\boldsymbol{n}}$-RGO series, which can be correlated with the smallest size of the starting dendrimer.

SEM and TEM analyses of $\mathbf{D G}_{\boldsymbol{n}}$-RGO reveal a similar morphology to $\mathbf{D G}_{\boldsymbol{n}}$-GO parent materials, although significant densification of layers and slight shrinkage of the network seem to occur during hydrazine treatment (Fig. 3a, b and S7a, ESI $\dagger$ ). HRTEM analyses show elongated core-shell crumbled nanostructures of several hundreds of nanometers in length with an interior width of 24 to $54 \mathrm{~nm}$ covered outside by a dense layer of 6 to $8 \mathrm{~nm}$ (Fig. 3d-f and S7b, ESI $\dagger$ ). Comparing the topology of $\mathbf{D G}_{\boldsymbol{n}}$-RGO and $\mathbf{D G}_{\boldsymbol{n}}$-GO, the variation concerns only the width and not the length of the elementary nanostructures. This reorganisation emanates probably from an interfacial folding of several elementary structures during hydrazine treatment; the reduction step is suspected to consume polar oxygenated groups and consequently minimise interfacial electrolytic complexation.

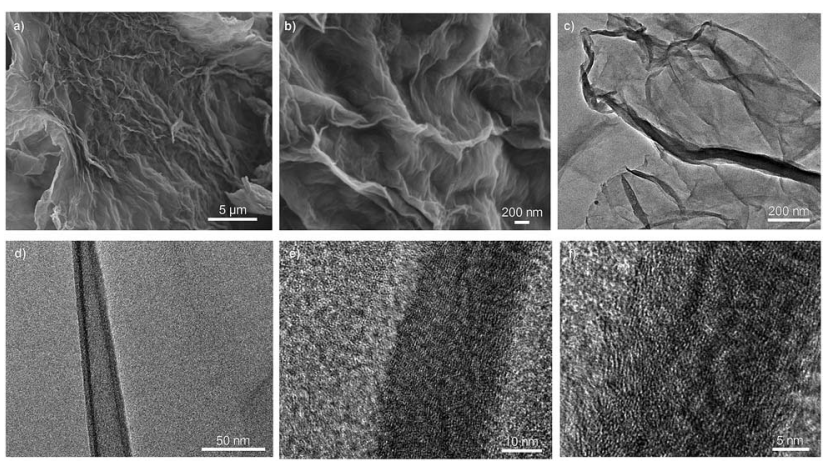

Fig. 3 Microscopic analysis of $\mathrm{DG}_{4}-\mathrm{RGO}$. (a and b) SEM of $\mathrm{DG}_{4}-\mathrm{RGO}$. (c and d) TEM and (e and f) HRTEM of DG H $_{4}$ RGO.

XRD reveals a slight decrease of the basal plane in $\mathbf{D G}_{\mathbf{1}}-\mathbf{R G O}$ (from $1.29 \mathrm{~nm}$ to $1.04 \mathrm{~nm}$ ) that can be explained by a decrease of the layer thickness during oxygen removal from the surface. The basal plane was still however high in $\mathbf{D G}_{\mathbf{1}}$-RGO compared to native GO $(d=0.91)$. The absence of any appreciable peak for DG $_{\boldsymbol{n}}$-RGO ( $n=2$ to 4 ) ruled out any restacking of graphene sheets and confirmed the total disordering and exfoliation of graphene sheets in these materials (Fig. S4, ESI $\dagger$ ).

\section{Chemical interplay at the $\mathrm{DG}_{\boldsymbol{n}}$-GO and $\mathrm{DG}_{\boldsymbol{n}}$-RGO interface}

The interfacial chemical interplay was accurately examined by coupling a set of analytical tools including RAMAN, X-ray photon spectroscopy (XPS) and solid-state NMR spectroscopy.

MAS CP ${ }^{13} \mathrm{C}$ NMR of $\mathbf{D G}_{\boldsymbol{n}}$-RGO revealed the first evidence for oxygen removal from the GO surface. A typical signal belonging to the graphene oxide at $\sim 70 \mathrm{ppm}$ completely disappeared as illustrated in the ESI (Fig. S3a, ESI $\dagger$ ). MAS CP ${ }^{31} \mathrm{P}$ NMR of the four materials displays the two typical signals of the core and the branching units (respectively at 4.8 to $7.5 \mathrm{ppm}$ and at 67 to $69 \mathrm{ppm}$ ) thereby indicating the intactness of the dendrimer skeleton during chemical reduction and precipitation-dispersion cycles (Fig. S3b, ESI $\dagger$ ).

We further investigated the variation observed in Raman spectroscopy, where the D (associated with the order/disorder of the system) and $\mathrm{G}$ (an indicator of the stacking structure) bands are the dominant vibrational modes observed respectively at 1354 and $1601 \mathrm{~cm}^{-1} \cdot{ }^{26,28}$ The ratio of the intensities of the two bands $(D / G)$ is often used to determine the overall stacking of the sheets, and high $\mathrm{D} / \mathrm{G}$ ratios indicate a high degree of exfoliation/disorder (Fig. S8, ESI $\dagger$ ). The intensity ratio of the starting GO increased from 1.15 to 1.25 when intercalated with $\mathbf{D G}_{\mathbf{1}}$ and to 1.36 for the full exfoliation reached in the case of the bulky $\mathbf{D G}_{\mathbf{4}}$-GO dendrimer. The highest exfoliation and disorder caused by $\mathbf{D G}_{\mathbf{4}}$ are further supported by the appearance of a new $\mathrm{D}^{\prime}$ band as a shoulder at $1619 \mathrm{~cm}^{-1}$ (Fig. 4). Upon reduction, a further increase of the $\mathrm{D} / \mathrm{G}$ ratio was observed for these materials compared to the starting $\mathbf{G O}$ (1.48 for $\mathbf{D G}_{\mathbf{1}}-\mathbf{R G O}, 1.54$ for $\mathbf{D G}_{\mathbf{2}}$-RGO, 1.58 for $\mathbf{D G}_{\mathbf{3}}$-RGO and 1.37 for $\mathbf{D G}_{\mathbf{4}}$-RGO) consistent with previous literature. ${ }^{26}$ Moreover, all these materials displayed an additional $\mathrm{D}^{\prime}$ band characteristic of increased disorder. Such perturbation, not observed in the case of 


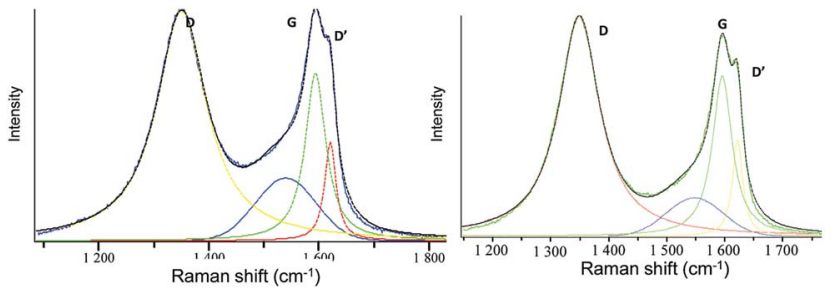

Fig. 4 RAMAN analysis of $D_{4}-G O$ (left) and $D G_{4}-R G O$ (right).

graphene oxide exfoliated ammonium-terminated chitosan, ${ }^{21}$ indicates that more disordering was experienced in the case of ternary ammonium-terminated dendrimers owing to their increased globular and radial (instead of linear) size bulkiness. The unique structure of phosphorus dendrimers and the highest ramification on the external surface maximise its contact with graphene sheets. The highest entropy reached under these circumstances allows for performing both parallel and perpendicular exfoliation depending on the thermodynamically favourable conformation..$^{29}$ The non-variation of the $\mathrm{D} / \mathrm{G}$ ratio in the special case of $\mathbf{D G}_{\mathbf{4}}$ before (1.36 for $\mathbf{D G}_{\mathbf{4}}-\mathbf{G O}$ ) and after hydrazine treatment ( 1.37 for $\mathbf{D G}_{\mathbf{4}}$-RGO) while keeping the $\mathrm{D}^{\prime}$ band indicates that the highest disorder was already reached during the dispersion step. The increased number of functional groups allows for the total consumption/binding of the dendrimer to the sheets and the significant wrapping of the graphene surface left few oxygenated groups accessible/available during hydrazine treatment. A negligible alteration (reduction) of the material surface was consequently observed in this case.

Insight into the $\mathbf{D G}_{\boldsymbol{n}}-\mathbf{G O}$ and $\mathbf{D} \mathbf{G}_{\boldsymbol{n}}-\mathbf{R G O}$ interfacial interplay was gained by XPS analyses. Pristine graphene oxide displays signals of carbon deconvoluted at $284.4(\mathrm{C}=\mathrm{C} / \mathrm{C}-\mathrm{C}), 286.5$ (C$\mathrm{O})$ and $288.5 \mathrm{eV}(-\mathrm{C}=\mathrm{O} / \mathrm{COO})$ and a minor $\pi-\pi^{*}$ plasmonic related band at $289.6 \mathrm{eV}$. The oxygen binding signal appears at $532.2 \mathrm{eV}$. We note the presence of an S (2p) doublet at 168.3$169.5 \mathrm{eV}$, attributed to sulfonic or sulfate groups generated during Hummer oxidation. In $\mathbf{D G}_{\boldsymbol{n}}$-GO $(n=1,4)$, binding energies of carbon, nitrogen, oxygen, sulfur and phosphorus

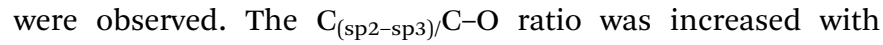
a decrease of the $\mathrm{COO}$ ratio and the $\pi-\pi^{*}$ indicative of a chemical reaction of peripheral ternary ammoniums with oxygenated groups as well as more disordering of carbon sheets because of their exfoliation (Table S1, ESI $\dagger$ ). Oxygen binding shows two oxygen types, $\mathrm{C}-\mathrm{O}$ and $\mathrm{C}-\mathrm{O}-\mathrm{P}$ bonding. Nitrogen also reveals two peaks at $398.9 \mathrm{eV}$ of $\mathrm{N}-\mathrm{P}$ and $\mathrm{C}-\mathrm{N}$ and at $401.2 \mathrm{eV}$ typical of quaternized nitrogen atoms. Sulfur (2p) spectra also confirm the presence of $\mathrm{P}=\mathrm{S}$ fragments belonging to the dendrimers at $161.3-162.5 \mathrm{eV}$ alone with residual sulfonate at higher binding energies (168.3-169.5 eV). Phosphorus binding reveals a broad signal corresponding to both $\mathrm{N}_{2} \mathrm{PO}$ and $\mathrm{N}_{4} \mathrm{PS}$ at 132.4 and $133.8 \mathrm{eV}$ (Fig. 5 and S9, ESI†). Lastly, chlorides (the counter ions of the ternary ammonium) remain on the material surface (with a binding energy of $197.0 \mathrm{eV}$ ) despite extensive washing of the material framework. However, its concentration is below the spectrometer sensitivity ( 0.1 at $\%)$.

Hydrazine treatment induces a significant alteration of the material-surface composition as substantiated by XPS and in full agreement with NMR and RAMAN analyses. Indeed, the C $1 \mathrm{~s}$ peak at $284.4 \mathrm{eV}$ becomes predominant, attributed mainly to carbon $\mathrm{sp}^{2}$, while $\mathrm{C}-\mathrm{O}$ peaks appear only as a shoulder with $\mathrm{C}-\mathrm{N}$ and $\mathrm{COO}$ consistent with the removal of oxygenated species from the surface. A significant change in the spectral shape of $O$ $1 \mathrm{~s}$ is noticed with $\mathrm{C}-\mathrm{O}-\mathrm{P}$ being more abundant compared to $\mathrm{C}-\mathrm{O}$ related bonds. In the meantime, a significant $\mathrm{NH}^{+}$related peak intensity decrease is observed alongside with the removal of chloride and sulphate species from the material surface during hydrazine treatment. The remanence of the phosphorus signal indicates the non-alteration of the phosphorus environment upon reduction. The atomic ratio of the chemical species is provided in the ESI section (Table S1, ESI $\dagger$ ) and the suggested mechanism is further discussed in detail (S10, ESI $\dagger$ ).

In UV-visible spectroscopy, significant to lower values were observed for $\mathbf{D G}_{\boldsymbol{n}}$ - $\mathbf{R G O}$ with respect to the parent $\mathbf{D G}_{\boldsymbol{n}} \mathbf{-} \mathbf{- G O}$
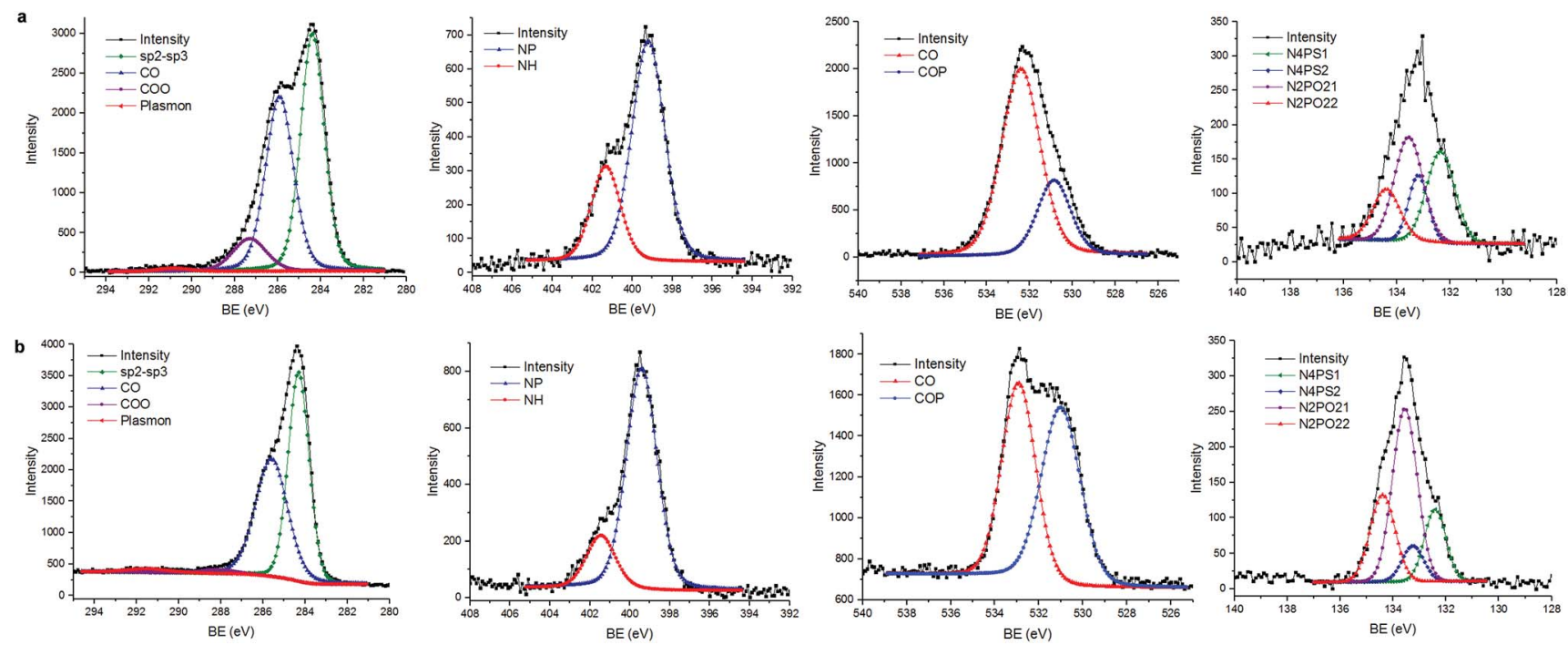

Fig. 5 XPS analyses (from left to right: binding energy of carbon, nitrogen oxygen and phosphorus elements) of (a) $D G_{4}-G^{-}$and (b) DG $-\mathrm{RGO}_{4}$ 


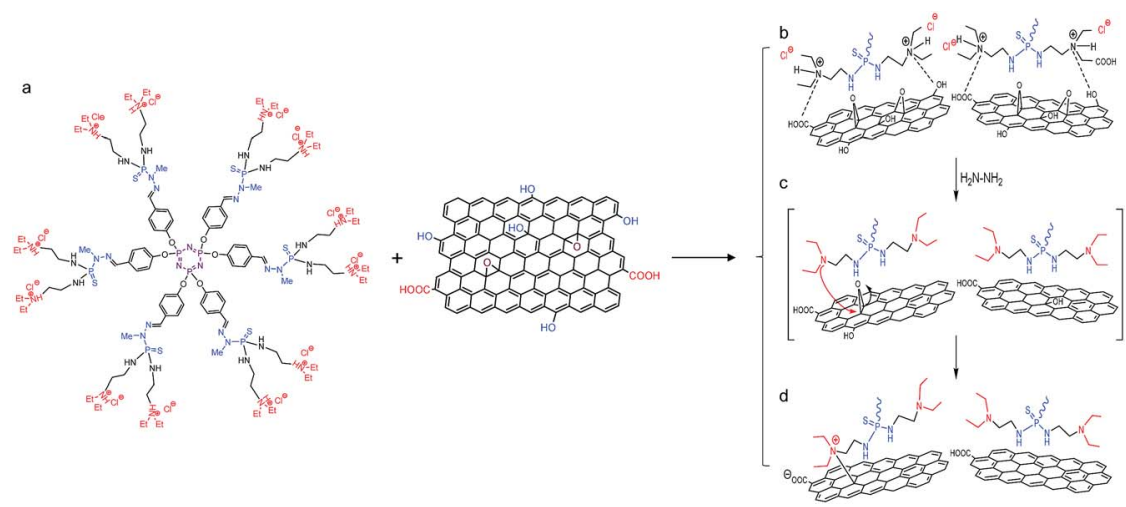

Scheme 1 Chemical interplay occurring at the dendrimer-graphene oxide interface. (a) Typical chemical structure of the first generation phosphorus dendrimer $\mathrm{DG}_{1}$ and oxygenated graphene oxide sheets. (b) Plausible mechanism occurring during interfacial complexation of ternary diethyl- $N$-amino-ammonium chloride arms and (c and d) the subsequent chemical reduction. See S10 in the ESI $\uparrow$ for further discussion.

materials (for instance from $290 \mathrm{~nm}$ to $285 \mathrm{~nm}$ and from $290 \mathrm{~nm}$ to $279 \mathrm{~nm}$ for $\mathbf{D G}_{2}-\mathbf{R G O}$ and $\mathbf{D G}_{3}-\mathbf{R G O}$, respectively). This shift indicates a significant alteration of the interfacial properties of $\mathbf{D G}_{\boldsymbol{n}}-\mathbf{R G O}$ compared to the starting $\mathbf{D G}_{\boldsymbol{n}}-\mathbf{G O}$ materials (Scheme 1). ${ }^{24}$

\section{${\mathrm{CS} @ D G_{n}-(\mathrm{R}) \mathrm{GO} \text { nanocomposite bioplastics }}$}

The stable exfoliation reached in these modified graphene sheets associated with their improved properties prompted their use as flame-retardant nano-fillers for soft-bioplastics. Among the various polymers suitable as film-forming matrices, we considered chitosan CS (a biodegradable glucosamine polymer derived from bio-waste generated in fishery plants, which is available in enormous quantity on earth) especially useful as a packaging material e.g. for healthcare, food and agriculture..$^{30}$

Our bioplastic was prepared in two steps starting first by solubilising medium molecular weight chitosan in aqueous acidic solution followed by the addition of $3 \mathrm{wt} \%$ of the filler $\left(\mathbf{G O}, \mathbf{D G}_{\boldsymbol{n}}\right.$-GO, and $\mathbf{D G}_{\boldsymbol{n}}-\mathbf{R G O}$ ) dispersed in aqueous solution. Casting of the mixture under air in a Petridish enables the formation of good-quality, crack-free films (Fig. 6). Interestingly, despite the bulkiness of the as-prepared nano-fillers, SEM analysis reveals excellent homogenisation of the film,

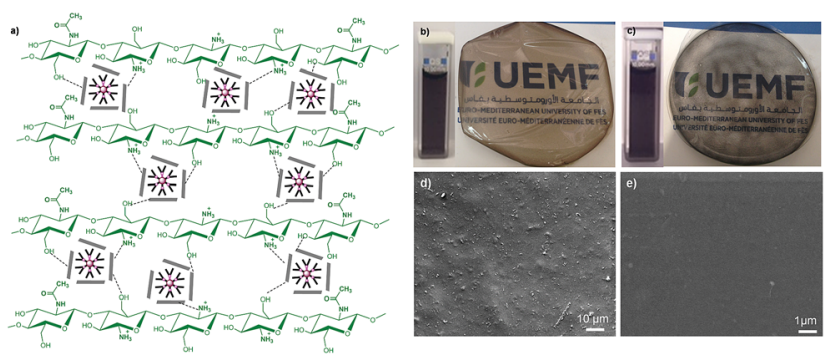

Fig. 6 (a) Intercalation of $\mathrm{DG}_{n}-\mathrm{RGO}$ within the fibrillary network of chitosan. (b) Aqueous dispersion of $\mathrm{DG}_{4}-\mathrm{GO}$ and the resulting transparent CS@DG - -GO film (top) and (d) the corresponding SEM analysis. (c) Aqueous dispersion of $\mathrm{DG}_{4}-\mathrm{RGO}$ and the resulting transparent $\mathrm{CS}_{\mathrm{ADG}}-\mathrm{RGO}$ film (top) and (e) the corresponding SEM analysis.

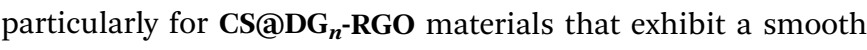
surface. No phase separation or filler aggregation was observed, which is an excellent preliminary result toward the rational design of functional, mechanically robust bioplastics.

Thermogravimetric analysis (TGA) allows monitoring the bioplastic degradation. Previously, we noticed the detrimental use of graphene oxide as a filler for bioplastics and evidenced that oxygenated groups catalytically accelerate the $\mathrm{C}-\mathrm{C}$ bond scission of the biopolymer. ${ }^{21}$ To our delight, we herein discovered that the degradation of chitosan was significantly delayed in the case of $\mathbf{C S} @ \mathbf{D G}_{\boldsymbol{n}}$-GO and even, substantial improvement

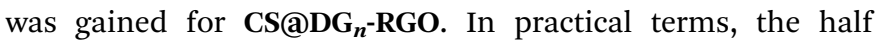
weight degradation of chitosan denoted as $\mathbf{T}_{\mathbf{5 0}}$ was measured to be $325{ }^{\circ} \mathrm{C}$. Improvement of $\mathbf{C S}$ thermal properties was noticed by the use of $\mathbf{D G}_{\mathbf{1}} \mathbf{-} \mathbf{G O}$ and $\mathbf{D G}_{\mathbf{4}}-\mathbf{G O}$ where $\mathbf{T}_{\mathbf{5 0}}$ was found to be $340{ }^{\circ} \mathrm{C}$ and $345{ }^{\circ} \mathrm{C}$, respectively. The performance was even pushed up for $\mathbf{C S} @ \mathbf{D G}_{4}-\mathbf{R G O}$ where its $\mathbf{T}_{50}$ reached nearly $400{ }^{\circ} \mathrm{C}$, corresponding to an impressive $\Delta T$ of $75{ }^{\circ} \mathrm{C}$ with respect to non-modified bioplastics (Fig. S11, ESI $\dagger$ ).

\section{Experimental}

\section{Synthesis of $\mathrm{DG}_{\boldsymbol{n}}$-GO and $\mathrm{DG}_{\boldsymbol{n}}$-RGO materials}

DG $_{n}$-GO. $40 \mathrm{mg}$ GO was dispersed in $100 \mathrm{~mL} \mathrm{H}_{2} \mathrm{O}$ by sonication and the dispersion was mixed with $\mathbf{D G}_{\boldsymbol{n}}(n=1,71 \mathrm{mg}$; $n=2,84 \mathrm{mg} ; n=3,93 \mathrm{mg} ; n=4,65 \mathrm{mg}$ ) and stirred at room temperature for $24 \mathrm{~h}$. The mixture was filtered and the solid was washed several times with water and ethanol and finally dried under vacuum at room temperature.

$\mathbf{D G}_{\boldsymbol{n}}$-RGO. $3 \mathrm{~mL}$ of hydrazine was added to a dispersion of $\mathbf{D G}_{n}-\mathbf{G O}(n=1,17 \mathrm{mg} ; n=2,25 \mathrm{mg} ; n=3,27 \mathrm{mg} ; n=4,22 \mathrm{mg})$ in $40 \mathrm{~mL} \mathrm{H}_{2} \mathrm{O}$. The mixture was stirred at room temperature for $24 \mathrm{~h}$. The mixture was filtered and the solid was washed several times with water and ethanol and finally dried under vacuum at room temperature.

\section{Conclusions}

In this contribution, high content heteroatoms (nitrogen, phosphorus, and sulfur) assembled in a tree-like dendritic 
network are confined within the layered galleries of graphene oxide. The globular structure of cation-terminated dendrimers enables them to act both as an exfoliating medium and a crosslinking agent to afford novel-extended nanostructures with highly disordered carbon sheets. Hydrazine treatment allowed substantial surface alteration of the ionic interfacial complexation without restacking of the graphene layers. Stable dispersion was obtained and as a proof of concept, the resulting functional ink was used as a nano-filler for aqueous-phase processing of bioplastics where significant improvement of the thermal stability was noticed. Considering the molecular diversity of $\mathbf{D G}_{\boldsymbol{n}}$-GO and $\mathbf{D G}_{\boldsymbol{n}}$-RGO materials, their synergistic functionality and the promising use of phosphorus dendrimers themselves in nanotechnology, these results appear promising for the development of multifunctional and biologically active flame-retardant hybrid mats and bioplastics.

\section{Conflicts of interest}

There are no conflicts to declare.

\section{Acknowledgements}

UEMF is warmly acknowledged for the financial support. We thank Vincent Collière for technical assistance (SEM and TEM analyses).

\section{Notes and references}

1 K. S. Novoselov, A. K. Geim, S. V. Morozov, D. Jiang, Y. Zhang, S. V. Dubonos, I. V. Grigorieva and A. A. Firsov, Science, 2004, 306, 666.

2 (a) M. Yi and Z. Shen, J. Mater. Chem. A, 2015, 3, 1170011715; (b) Y. L. Zhong, Z. Tian, G. P. Simon and D. Li, Mater. Today, 2015, 18, 73-78.

3 (a) V. Georgakilas, J. N. Tiwari, K. C. Kemp, J. A. Perman, A. B. Bourlinos, K. S. Kim and R. Zboril, Chem. Rev., 2016, 116, 5464-5519; (b) J. E. Johns and M. C. Hersam, Acc. Chem. Res., 2013, 46, 77-86; (c) M. Quintana, E. Vazquez and M. Prato, Acc. Chem. Res., 2013, 46, 138-148.

4 K. Hu, D. D. Kulkarni, I. Choi and V. V. Tsukruk, Prog. Polym. Sci., 2014, 39, 1934-1972.

5 J.-Y. Liu, X.-X. Li, J.-R. Huang, J.-J. Li, P. Zhou, J.-H. Liu and X.-J. Huang, J. Mater. Chem. A, 2017, 5, 5977-5994.

6 (a) S. Navalon, A. Dhakshinamoorthy, M. Alvaro and H. Garcia, Chem. Rev., 2014, 114, 6179-6212; (b) S. Navalon, A. Dhakshinamoorthy, M. Alvaro, M. Antonietti and H. Garcia, Chem. Soc. Rev., 2017, 46, 4501-4529.

7 (a) R. Zhang and W. Chen, Biosens. Bioelectron., 2017, 89(Part 1), 249-268; (b) A. Ambrosi, C. K. Chua, A. Bonanni and M. Pumera, Chem. Rev., 2014, 114, 7150-7188.

8 (a) K. Yang, L. Feng, X. Shi and Z. Liu, Chem. Soc. Rev., 2013, 42, 530-547; (b) C. Cheng, S. Li, A. Thomas, N. A. Kotov and R. Haag, Chem. Rev., 2017, 117, 1826-1914.

9 (a) S. Ravula, S. N. Baker, G. Kamath and G. A. Baker, Nanoscale, 2015, 7, 4338-4353; (b) A. Ciesielski and
P. Samori, Adv. Mater., 2016, 28, 6030-6051; (c) J. I. Paredes and S. Villar-Rodil, Nanoscale, 2016, 8, 15389-15413.

10 D. R. Dreyer, S. Park, C. W. Bielawski and R. S. Ruoff, Chem. Soc. Rev., 2010, 39, 228-240.

11 (a) P. T. Yin, S. Shah, M. Chhowalla and K.-B. Lee, Chem. Rev., 2015, 115, 2483-2531; (b) T. Premkumar and K. E. Geckeler, Prog. Polym. Sci., 2012, 37, 515-529; (c) X. Wang, E. N. Kalali, J.-T. Wan and D.-Y. Wang, Prog. Polym. Sci., 2017, 69, 22-46; (d) R. Zappacosta, M. Di Giulio, V. Ettorre, D. Bosco, C. Hadad, G. Siani, S. Di Bartolomeo, A. Cataldi, L. Cellini and A. Fontana, J. Mater. Chem. B, 2015, 3, 6520-6527; (e) J. Suarez-Guevara, V. Ruiz and P. Gomez-Romero, Phys. Chem. Chem. Phys., 2014, 16, 20411-20414.

12 (a) Y. Brahmi, N. Katir, J. A. M. Agullo, A. Primo, M. Bousmina, J. P. Majoral, H. Garcia and A. El Kadib, Dalton Trans., 2015, 44, 15544-15556; (b) K. Milowska, A. Rybczyńska, J. Mosiolek, J. Durdyn, E. M. Szewczyk, N. Katir, Y. Brahmi, J.-P. Majoral, M. Bousmina, M. Bryszewska and A. El Kadib, ACS Appl. Mater. Interfaces, 2015, 7, 19994-20003; (c) Y. Brahmi, N. Katir, M. Ianchuk, V. Colliere, E. M. Essassi, A. Ouali, A.-M. Caminade, M. Bousmina, J. P. Majoral and A. El Kadib, Nanoscale, 2013, 5, 2850-2856.

13 (a) A. El Kadib, N. Katir, M. Bousmina and J. P. Majoral, New J. Chem., 2012, 36, 241-255; (b) A.-M. Caminade and J.-P. Majoral, Chem. Soc. Rev., 2010, 39, 2034-2047.

14 (a) D. A. Tomalia, H. Baker, J. Dewald, M. Hall, G. Kallos, S. Martin, J. Roeck, J. Ryder and P. Smith, Macromolecules, 1986, 19, 2466-2468; (b) R. Roy and T. C. Shiao, Chem. Soc. Rev., 2015, 44, 3924-3941; (c) A. Sheikhi, N. Li, T. G. M. van de Ven and A. Kakkar, Environ. Sci.: Water Res. Technol., 2016, 2, 71-84; (d) N. Katir, N. El Brahmi, A. El Kadib, S. Mignani, A.-M. Caminade, M. Bousmina and J. P. Majoral, Chem.-Eur. J., 2015, 21, 6400-6408; (e) A.-M. Caminade, A. Ouali, R. Laurent, C.-O. Turrin and J.-P. Majoral, Chem. Soc. Rev., 2015, 44, 3890-3899.

15 (a) A.-M. Caminade, A. Hameau and J.-P. Majoral, Dalton Trans., 2016, 45, 1810-1822; (b) C.-O. Turrin, V. Maraval, J. Leclaire, E. Dantras, C. Lacabanne, A.-M. Caminade and J.-P. Majoral, Tetrahedron, 2003, 59, 3965-3973.

16 (a) A.-M. Caminade, R. Laurent, C.-O. Turrin, C. Rebout, B. Delavaux-Nicot, A. Ouali, M. Zablocka and J.-P. Majoral, C. R. Chim., 2010, 13, 1006-1027; (b) N. Katir, N. El Brahmi, N. Marcotte, J. P. Majoral, M. Bousmina and A. El Kadib, Macromolecules, 2016, 49, 5796-5805.

17 J. Leclaire, Y. Coppel, A.-M. Caminade and J.-P. Majoral, J. Am. Chem. Soc., 2004, 126, 2304-2305.

18 (a) K. Ciepluch, N. Katir, A. El Kadib, M. Weber, A.-M. Caminade, M. Bousmina, J. Pierre Majoral and M. Bryszewska, J. Lumin., 2012, 132, 1553-1563; (b) K. Ciepluch, M. Weber, N. Katir, A.-M. Caminade, A. El Kadib, B. Klajnert, J. P. Majoral and M. Bryszewska, Int. J. Biol. Macromol., 2013, 54, 119-124.

19 N. Katir, A. El Kadib, V. Colliere, J. P. Majoral and M. Bousmina, Chem. Commun., 2014, 50, 6981-6983. 
20 (a) N. Katir, Y. Brahmi, J. P. Majoral, M. Bousmina and A. El Kadib, Chem. Commun., 2015, 51, 17716-17719; (b) Y. Brahmi, N. Katir, A. Hameau, A. Essoumhi, E. M. Essassi, A.-M. Caminade, M. Bousmina, J.-P. Majoral and A. El Kadib, Chem. Commun., 2011, 47, 8626-8628.

21 S. Frindy, A. Primo, H. Ennajih, A. el kacem Qaiss, R. Bouhfid, M. Lahcini, E. M. Essassi, H. Garcia and A. El Kadib, Carbohydr. Polym., 2017, 167, 297-305.

22 C. Loup, M. A. Zanta, A. M. Caminade, J. P. Majoral and B. Meunier, Chem.-Eur. J., 1999, 5, 3644-3650.

23 J. Zou and F. Kim, ACS Nano, 2012, 6, 10606-10613.

24 F. T. Johra, J.-W. Lee and W.-G. Jung, J. Ind. Eng. Chem., 2014, 20, 2883-2887.
25 J. Zou and F. Kim, Nat. Commun., 2014, 5, 5254.

26 S. Stankovich, D. A. Dikin, R. D. Piner, K. A. Kohlhaas, A. Kleinhammes, Y. Jia, Y. Wu, S. T. Nguyen and R. S. Ruoff, Carbon, 2007, 45, 1558-1565.

27 D. A. C. Brownson, D. K. Kampouris and C. E. Banks, Chem. Soc. Rev., 2012, 41, 6944-6976.

28 M. S. Dresselhaus, A. Jorio, A. G. Souza Filho and R. Saito, Philos. Trans. R. Soc. London, Ser. A, 2010, 368, 5355-5377.

29 J. Yang, X. Yang and Y. Li, Curr. Opin. Colloid Interface Sci., 2015, 20, 339-345.

30 (a) M. Aider, Food Sci. Technol., 2010, 43, 837-842; (b) M. Z. Elsabee and E. S. Abdou, Mater. Sci. Eng., C, 2013, 33, 1819-1841. 